\title{
Advances in Nucleotide Antiviral Development from Scientific Discovery to Clinical Applications: Tenofovir Disoproxil Fumarate for Hepatitis B
}

\author{
Lillian Lou
}

Nexus Development PA

\begin{abstract}
Exploration of naturally occurring chemical structures for medicinal uses has received significant interest in drug discovery and development research in the past few decades. None have had more success or products of greater clinical efficacy than synthetic analogs of nucleosides and nucleotides, especially as antiviral drugs. Nucleos(t)ide antivirals are synthetic analogs of the natural building blocks of DNA or RNA. This review focuses on the developmental path of tenofovir disoproxil fumarate (TDF), a prodrug of a nucleotide analog and its clinical applications as a first-line antiviral for chronic hepatitis $B(\mathrm{CHB})$.

Tenofovir is a potent antiviral compound, but has poor oral availability. The disoproxil fumarate (DF) prodrug moiety greatly enhances intestinal absorption allowing it to become an oral medication. Tenofovir is activated intracellularly, and the incorporation into HBV DNA prevents further elongation thus terminating replication. In patients with $\mathrm{CHB}$, TDF has demonstrated broad, potent and sustained virologic response. Maintenance of viral suppression for up to 5 years resulted in regression of fibrosis and cirrhosis. No tenofovirresistant HBV variants have been detected in patients after long-term use. The efficacy and safety profiles reported from cohort studies of clinical practices were consistent with those observed in registration trials.

Continuous development includes a new oral prodrug, tenofovir alafenamide fumarate (TAF), which has enhanced delivery of tenofovir to target cells compared to TDF.

(c) 2013 The Second Affiliated Hospital of Chongqing Medical University. Published by XIA \& HE Publishing Ltd. All rights reserved.
\end{abstract}

\section{Introduction}

Nucleotide analog drugs have advantages over nucleosides as the former bypass the first of three phosphorylation steps, often the most challenging gate-keeping requirement for activation in cells. In addition, nucleotides are negatively

Keywords: Nucleotide antiviral; Tenofovir; Prodrug; Hepatitis B.

Abbreviations: TDF, tenofovir disoproxil fumarate; $\mathrm{CHB}$, chronic hepatitis $\mathrm{B}$; $D F$, disoproxil fumarate; TAF, tenofovir alafenamide fumarate; dATP, deoxyadenosine triphosphate; LAM, lamivudine; ETV, Entecavir; HBIg, hepatitis B immunoglobulin.

Received: 21 January 2013; Revised: 9 March 2013; Accepted: 15 March 2013 DOI of original article: http://dx.doi.org/10.14218/JCTH.2013.004XX.

Correspondence to: Lillian Lou, PO Box 1345, Palo Alto, CA 94302, USA. Tel.: 650-245-7094, E-mail: LLou@NexusDevPA.com charged so they have extended intracellular retention compared to the nucleosides. The nucleotide analogs are not simple chemical derivatives of natural metabolites as the nucleobase portion of the molecules is not modified. However, the sugar and phosphate portions are drastically altered from the natural structures. Medicinal chemists in collaboration with virologists have invented biologically active molecules by considering the steric and electronic properties of nucleotides, and by synthesizing series of analogs for testing. The landmark publication in 1986 by Erik De Clercq and Antonin Holý suggested the tremendous potential for the acyclic nucleotide analogs such as tenofovir as antiretroviral agents. $^{1}$

TDF (Viread ${ }^{\mathbb{R}}$; Gilead) has been approved for the treatment of HIV-1 infection in combination with other antiretrovirals since 2001, and for CHB as monotherapy since 2008. In the most recent updates, approval was extended to include pediatric HIV patients of 2 years and older, and $\mathrm{CHB}$ patients of 12 years and older. ${ }^{2-4}$ Currently, TDF is licensed for the treatment HIV/AIDS in 103 countries and for CHB in 56 countries worldwide. Although not the first nucleotide antiviral to be licensed, TDF is commonly recommended as a first-line drug for $\mathrm{CHB} .{ }^{5-7}$ TDF-containing regimens especially those in fixed dose combination with emtricitabine (FTC) have become the most preferred backbone of first-line antiretroviral therapies. ${ }^{8,9}$ The fixed dose combination of FTC/TDF (Truvada ${ }^{\circledR}$; Gilead) was recently granted approval by the US FDA for pre-exposure prophylaxis to reduce the risk of sexually acquired HIV-1 in high-risk adults. ${ }^{10,11}$ This present review focuses on the past and ongoing development of TDF and its clinical applications in patients with CHB. This manuscript is intended to provide an overview rather than a comprehensive review of the clinical literature on the treatment of CHB with TDF.

\section{Delivery of tenofovir and activation in target cells}

TDF is a prodrug of tenofovir, an acyclic nucleoside-phosphonate (nucleotide) analog of deoxyadenosine monophosphate (dAMP). Tenofovir has been shown to be a potent antiviral compound against HIV and HBV in cell cultures. However, it is poorly absorbed after oral administration presumably due to the negative charges on the phosphonyl group. Through structural optimization and laboratory testing of a series of chemical candidates, a novel DF prodrug moiety added to tenofovir was found to greatly enhance its intestinal absorption and uptake into target cells. ${ }^{12-14}$ In preclinical studies, oral administration of TDF delivered more tenofovir 
than subcutaneous injection of tenofovir. ${ }^{15}$ The DF prodrug innovation and the long intracellular half-life $(12-15 \mathrm{~h}$ in activated and $\sim 50 \mathrm{~h}$ in resting lymphocytes) ${ }^{14}$ of tenofovir allowed it to become a once daily oral medication. A convenient dosing schedule is especially favorable for adherence in patients being treated for chronic infection. Once absorbed into the circulation, the prodrug moiety is rapidly removed by serum esterases to produce tenofovir. Both TDF and tenofovir can be taken up by target cells. Tenofovir is converted through cellular phosphorylation by host enzymes to the pharmacologically active metabolite tenofovir-diphosphate (DP), which is an analog of deoxyadenosine triphosphate (dATP) (Fig. 1), ${ }^{14}$ a natural precursor of DNA. Unlike human polymerases, HBV and other viral reverse transcriptase/polymerases (rt/pol) are less specific in selecting the correct nucleotide precursors for building DNA. This error-prone characteristic of the viral enzymes forms the basis of developing drug-resistance through mutations, but also makes it possible for certain synthetic analogs to substitute for the naturally existing precursors during viral replication. Because of this promiscuous property, HBV rt/pol recognizes tenofovir-DP, and allows it to compete with the cognate dATP and utilize tenofovir-DP as a building block in place of dATP for viral DNA synthesis. Once incorporated, tenofovir prevents the HBV DNA chain for further elongation because the structure of tenofovir renders it chemically impossible to incorporate subsequent incoming nucleotides to the growing viral DNA. This chain-terminating mechanism of action is highly effective in preventing viral replication. Tenofovir-DP binds to HBV rt/pol at the enzyme active site, whose structure is conserved between viral genotypes, and is reflected in the broad genotypic activity of TDF. ${ }^{16}$

\section{TDF superseded Adefovir dipivoxil}

Adefovir dipivoxil (ADV, Hepsera ${ }^{\mathbb{R}}$; Gilead) was the first nucleotide analog approved for the treatment of $\mathrm{CHB}$. In the initial stages of development, ADV was investigated for treatment of HIV/AIDS. Adefovir required a prodrug to improve oral absorption and chemical optimization resulted in ADV (dipivoxil ester). However, at the high doses investigated for
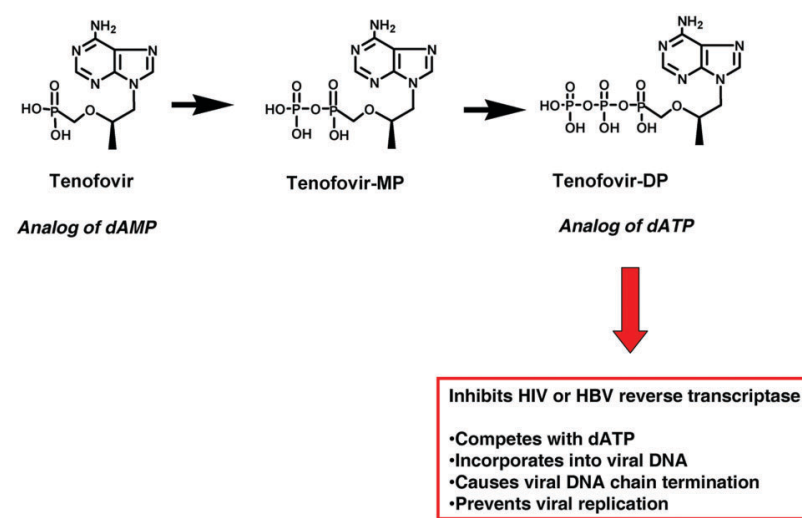

Fig. 1. A scheme depicting intracellular activation, and the antiviral mechanism of action of tenofovir. After transport into cells, tenofovir is phosphorylated in two steps by host nucleotide kinases to tenofovir-monophosphate (MP) and then tenofovir-DP, the active metabolite. Tenofovir inhibits viral replication by mechanisms indicated in the box.
HIV/AIDS, the particular prodrug group caused depletion of serum carnitine so it was necessary for patients to receive daily carnitine supplement concomitantly. ${ }^{17}$ More importantly, during this stage, unexpected renal safety concerns became apparent at doses of $60 \mathrm{mg}$ and $120 \mathrm{mg}$ in patients infected with HIV. These findings triggered the withdrawal of the New Drug Application to the US FDA in 1999. Kahn and colleagues reported that after 24 weeks of treatment, $120 \mathrm{mg}$ of ADV taken daily was associated with delayedonset of renal toxicity manifested by serum creatinine elevation or hypophosphatemia in HIV-infected patients. ${ }^{18}$ Kaplan-Meier analysis estimated $35 \%$ of patients would develop serum creatinine elevation of more than $0.5 \mathrm{mg} / \mathrm{dL}$ from baseline by week 48 and $50 \%$ by week 72 .

ADV was found to be more effective for treating HBV than HIV infection, and was studied at lower doses. In phase 2 studies in patients with $\mathrm{CHB}$, daily doses of $30 \mathrm{mg}$ or above were more efficacious than $10 \mathrm{mg} .{ }^{19}$ However, the higher doses were associated with an increased frequency of adverse events and renal laboratory abnormalities, while the $10 \mathrm{mg}$ dose showed a favorable safety profile. ${ }^{20}$ ADV at $10 \mathrm{mg}$ was approved in 2002 based on two pivotal studies demonstrating favorable efficacy, safety and resistance profile for the treatment of $\mathrm{CHB} .{ }^{20,21}$ ADV had a better drug resistance profile than lamivudine (LAM); it was also found to be effective for treating patients who developed resistance to LAM and, therefore, fulfilled an important unmet need at that time.

ADV has now been superseded by TDF primarily because the latter has superior efficacy at the approved dose $(300 \mathrm{mg})$, and better safety and resistance profiles. Of interest, tenofovir and adefovir are differentiated structurally by the critical placement of a pivotal methyl group, which defines virological and biological activities. Although the two are similar in antiviral potency in cell culture systems, they are distinct based on their recognition by the human mitochondrial DNA polymerase $\gamma(\mathrm{pol}-\gamma)$. In a biochemical analysis, tenofovir-DP was minimally incorporated by pol- $\gamma$ into mitochondrial DNA $(0.06 \%$ versus the natural nucleotide) while adefovir-diphosphate had 100 times more incorporation ( $6 \%$ versus the natural nucleotide). ${ }^{22}$ For comparison, LAM-triphosphate was incorporated at $0.13 \%$. This propensity towards interference with mitochondrial DNA synthesis might be, in part, related to the associated, but low level, renal safety concerns of ADV during long-term treatment. ${ }^{23}$ The structural differences of TDF compared to ADV provide the former with a more favorable efficacy, safety and resistance profile.

\section{TDF in treatment-näive CHB patients}

Head-to-head comparison with ADV and multi-year follow up

TDF registration was based on two double-blind, randomized, phase 3 trials comparing TDF with ADV for 48 weeks. Viral suppression (reduction of HBV DNA to less than 400 copies/ $\mathrm{mL}$ ) occurred in more HBeAg-negative (93\% vs. 63\%) and HBeAg-positive patients (76\% vs. $13 \%$ ) receiving TDF than patients receiving ADV. ${ }^{16}$ Baseline viral load was lower in $\mathrm{HBeAg}$-negative than $\mathrm{HBeAg}$-positive patients reflecting the ability of the former to achieve viral suppression after 48 weeks. HBeAg seroconversion rates were $21 \%$ for TDF, and $18 \%$ for ADV. HBsAg loss after 48 weeks with TDF occurred in $3 \%$ of patients who were $\mathrm{HBeAg}$-positive at baseline. This 
rate is the highest among anti-HBV nucleos(t)ides, and was comparable to that of pegylated interferon. In contrast, no patients receiving ADV experienced HBsAg loss. Nausea was the only adverse event that consistently occurred more frequently in patients receiving TDF than ADV. Among 641 patients (426 TDF; $215 \mathrm{ADV}$ ), only 1 patient, who received $A D V$, had confirmed serum creatinine elevation of more than $0.5 \mathrm{mg} / \mathrm{dL}$ from baseline. No patient developed resistance to tenofovir, while 1 patient developed the signature substitution rtN236T resistance to adefovir, and 3 patients developed an rtA181T substitution in the background of an rtM204I (signature LAM-resistance) mutation.

Patients who completed the double-blind phase of the phase 3 trials continued with an open-label phase receiving TDF. Of the 641 patients who received randomized treatment, $585(91 \%)$ entered the open-label phase and 489 (76\%) completed 240 weeks. The multi-year follow-up design, and high rate of patient retention allowed evaluation of the safety and efficacy of TDF treatment in a large cohort of $\mathrm{CHB}$ patients over several years. The open-label phase is still ongoing and is planned to continue for a total of 9 years. The treatment with TDF from week 48 onward for all enrolled patients provided the two important observations: 1) suboptimal response with ADV was rapidly circumvented with switching to TDF; 2) addition of FTC was not more efficacious than TDF alone in those patients who remained viremic, and took the option to add FTC after 72 weeks. ${ }^{24}$ At week 240, $75 \%$ of patients in the intent-to-treat analysis had HBV less that 400 copies $/ \mathrm{mL}$. Of those patients on treatment, $98 \%$ had HBV DNA less than 400 copies $/ \mathrm{mL}$, and $49 \%$ and $40 \%$ had $\mathrm{HBeAg}$ loss and seroconversion, respectively. HBsAg loss and seroconversion was $10 \%$ and $8 \%$, respectively in patients who were $\mathrm{HBeAg}$-positive at baseline based on Kaplan-Meier estimation. Only one patient $(<1 \%)$ who was HBeAgnegative at baseline had HBsAg seroconversion by week 240. TDF treatment was well tolerated. Nine (2\%) patients experienced treatment-related serious adverse events over the 240 week duration. Eight (1\%) discontinuations were due to adverse events. Laboratory abnormalities including those indicative of renal events were infrequent during the 240 week. ${ }^{25}$

Resistance testing for HBV rt/pol gene sequence mutations was conducted on the small number of patients who were viremic at every 48 week time point after treatment initiation, experienced viral breakthrough during the study period or who had an early discontinuation while viremic. No mutations associated with resistance to tenofovir were detected during 240 weeks of treatment. ${ }^{25}$ These data suggest that TDF's high barrier to genotypic resistance and potent antiviral efficacy may together result in durable viral suppression.

To date, the effectiveness of TDF in treating $\mathrm{CHB}$ patients in everyday clinical practice has been reported in four real-life cohorts in Europe. ${ }^{26-28} \mathrm{High}$ rate of durable viral suppression, absence of drug resistance, and favorable tolerability were reported, and were consistent with the results of the registration trials.

\section{Clinical benefit of long-term viral suppression}

In small subsets of patients, it has previously been reported that reversal of fibrosis and cirrhosis occurred after prolonged treatment with anti-HBV agents. In the TDF phase 3 studies, 348 patients had liver biopsy results at both baseline and

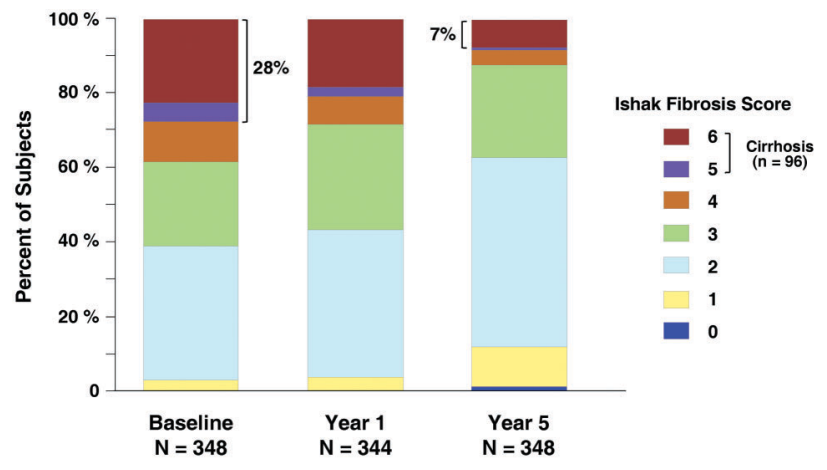

Fig. 2. Regression of liver fibrosis over 5 years of treatment with TDF. Distribution of Ishak fibrosis scores is shown for 348 patients with baseline and year 5 data, and 344 with data for all three time points. ${ }^{25}$

week 240. Marcellin and colleagues reported that 304 (87\%) of the 348 patients had histological improvement, and 176 $(51 \%)$ had regression of fibrosis at week $240(P<0.0001)$. Fig. 2 shows the time course of histologic improvement over 5 years. $^{25}$

At baseline, 96 (28\%) of 348 patients had cirrhosis (Ishak score 5 or above). At week 240, 71 (74\%) had a reduction in fibrosis, and no longer had cirrhosis. All, but one had a reduction of Ishak scores by 2 units or more. Three of 252 patients who had no cirrhosis at baseline progressed to cirrhosis at year 5 .

Nearly all patients who participated in the histology analysis achieved viral suppression. This significant histologic improvement observed in such large sample size with 5 years of TDF treatment definitively supports the notion that longterm viral suppression should be the treatment goal for antiHBV therapeutics.

\section{TDF in CHB patients previously treated with nucleos(t)ides}

Effectiveness of TDF for the treatment of LAM-resistant hepatitis B was recognized in HIV/HBV co-infection before regulatory approval was granted for $\mathrm{CHB}^{29-31}$ Similar to treatment-naïve patients, TDF has higher antiviral activity for treating LAM-resistant hepatitis B than ADV. ${ }^{32-34}$

The nucleoside antivirals LAM and telbivudine (LdT) have high propensities to select for HBV rt/pol variants highly resistant to the drugs. Some of the most common substitutions resistant to LAM, rtM204V and rtL180M, are also resistant to LdT. Entecavir (ETV) has a high barrier for developing genotypic resistance, except if LAM-resistant substitutions are pre-existing, such as in patients who failed LAM treatment or were infected with LAM-resistant HBV. The LAM-resistant substitutions can serve as a trigger, lowering ETV's barrier, to readily select for ETV resistance. Fortunately, HBV variants resistant to LAM, LdT and ETV are all fully susceptible to tenofovir. 6,35

Fung and colleagues recently reported a prospective, randomized, double-blind study comparing TDF with the FTC/TDF combination in patients with documented LAMresistant substitutions and who were viremic at baseline. It should be noted that FTC/TDF fixed-dose combination is not an FDA-approved treatment of CHB. After 96 weeks, viral suppression (HBV DNA $<400$ copies $/ \mathrm{mL}$ ) was reached in $89 \%$ of patients treated with TDF, and $86 \%$ with the 
combination. Both treatments were well tolerated with favorable safety profiles. There were no clinically significant changes in renal function, and no evidence of clinically relevant bone loss through the treatment period. ${ }^{34}$ Another randomized, placebo-controlled study enrolled adolescent patients with $\mathrm{CHB}$ including two-thirds of whom had prior exposure to LAM or ADV. At week 72, $89 \%$ of patients who received TDF and none on placebo reached virologic suppression. ${ }^{4}$ Increases in bone mineral density were significantly less in the TDF-treated than the placebo group. Bone safety with long-term TDF use in adolescents is not well understood and monitoring will continue. ${ }^{4}$

Adefovir-resistant variants, especially the double substitution rtN236T \pm rtA181T/V, have slightly reduced susceptibility to tenofovir in cell-based studies. ${ }^{6,35,37}$ Several studies in patients who failed treatments with ADV and/or LAM showed that TDF was effective in viral suppression; the presence of baseline mutations conferring resistance to LAM and/or adefovir did not influence treatment response. ${ }^{38-42}$ However, variability in the time to virologic response was apparent.

In a randomized, double-blind study in CHB patients who remained viremic after treatment with ADV (58\% had previous treatment with LAM), compared TDF and FTC/TDF. At week $48,81 \%$ from both groups had HBV DNA less than 400 copies/mL; inclusion of FTC had no added benefit. ${ }^{38}$ In a prospective open-label multicenter study that enrolled suboptimal responders receiving ADV \pm LAM, $48 \%$ and $55 \%$ had substitutions resistant to adefovir and LAM, respectively. ADV was replaced by TDF as mono- or add-on therapy according to the regimen at enrollment for individual patients, and after 48 weeks, $82 \%$ reached HBV DNA levels less than $69 \mathrm{IU} / \mathrm{mL} .{ }^{39} \mathrm{~A}$ retrospective study was conducted to assess the effectiveness of TDF in patients with prior failure or resistance to antiHBV nucleos(t)ides. After 23 months, $100 \%$ of patients with LAM-resistance or suboptimal response to ADV without genotypic adefovir-resistance reached HBV DNA levels $<400$ copies $/ \mathrm{mL}$, while only $52 \%$ of those with genotypic adefovir-resistant substitutions at baseline reached the virologic endpoint. However, within the adefovir-resistant group, the patients with lower viral load at baseline had an increased chance of reaching the endpoint. ${ }^{40} \mathrm{~A}$ prospective study evaluated TDF rescue therapy in heavily-pretreated CHB patients who had prior failure with LAM and remained viremic while receiving ADV. Only 64\% reached HBV DNA less than $15 \mathrm{IU} / \mathrm{ml}$ after 96 weeks of TDF treatment. However, continuous follow-up showed that $81 \%$ (per protocol) reached the endpoint after 5 years. ${ }^{41,42}$ Although there was no association between virologic response and adefovirresistant substitutions at baseline, patients who harbored the rtN236T adefovir-resistant substitution tended to have slower rates of viral decline on TDF than those without rtN236T. It was also noted that the patients with adefovirresistant substitutions had higher viral loads at baseline. ${ }^{41,42}$

These results taken together indicate that higher baseline viral loads might be associated with slower declines in DNA levels in those with adefovir-resistance. Other factors in addition to the changes at the HBV rt/pol sequences, may work in concert to define the evolution, selection and suppression of the resistant variants by antivirals. To understand why TDF has good efficacy in many patients with adefovir resistance, but has diminished efficacy in others will likely require a deeper understanding of host and viral interactions.

\section{TDF for CHB patients with severe liver disease}

The efficacy of TDF in CHB was first reported in patients suffering from LAM-resistant hepatitis B. In the early 2000's, clinical investigators noticed that TDF lowered HBV viral loads in patients co-infected with HIV/HBV who developed LAMresistant hepatitis $B$ while receiving LAM-containing antiretroviral regimens. ${ }^{29-31}$ Also, during this time, TDF and ADV were used as new rescue agents for patients who had HBV breakthroughs while receiving LAM \pm hepatitis B immunoglobulin (HBIg) post-liver transplantation. ${ }^{43}$

Prevention of hepatitis B recurrence post-liver transplantation was further confirmed in a randomized controlled study. Transplant recipients who were stable on HBIg prophylaxis were enrolled to receive FTC/TDF plus HBIg for 24 weeks, and then randomized to continue the dual therapy or receive only FTC/TDF. Final results have shown no recurrence of hepatitis $B$ up to 72 weeks after randomization with or without HBIg. ${ }^{44}$ FTC/TDF was well tolerated.

Safety and tolerability of TDF in patients with decompensated CHB liver disease was assessed in a randomized, double-blind study. Interim results at week 48 showed good virologic response, and more importantly, that adverse events and laboratory profiles were consistent with advanced liver disease and complications, with no unexpected safety signals. ${ }^{45}$

For more than a decade, the number of patients on lists waiting to receive liver transplants has been decreasing in the US. Especially noticeable is the rapid decline by $47 \%$ from 1999 to 2006 of incidence rate of waiting list registration for end stage liver disease due to hepatitis B compared to that due to hepatitis $C$ or non-viral liver disease. ${ }^{46}$ The temporal correlation of the trend and the availability of anti-HBV nucleos(t)ides, starting with use of LAM in 1998, suggests that broad application of these medications, in part, contributed to the decreased incidence of decompensated CHB disease.

\section{Tenofovir alafenamide fumarate - a new tenofovir prodrug}

The TDF prodrug enhanced oral absorption and allowed tenofovir to be dosed conveniently by mouth. The prodrug is also resulted in better cellular uptake than unmodified tenofovir. However, the DF prodrug moiety is rapidly hydrolyzed by serum esterases leaving tenofovir in the circulation. ${ }^{47}$ Continuing medicinal research aimed to improve cellular delivery of tenofovir by decreasing prodrug susceptibility to serum esterases in the circulation, and increasing conversion to tenofovir once inside cells. Tenofovir alafenamide fumarate (TAF) or GS7340 is a new prodrug of tenofovir selected for these properties, and is currently undergoing clinical investigation for HIV/AIDS and CHB.

TAF is more stable than TDF in circulation, and yet is more rapidly converted to tenofovir inside target cells (Fig. 3). ${ }^{47}$ A recent dose-ranging study in patients with HIV-infection showed that $8 \mathrm{mg}$ of TAF produced the same, and $25 \mathrm{mg}$ produced more than 7 times the active metabolite tenofovirDP in PBMCs as $300 \mathrm{mg}$ of TDF. The more efficient delivery of the active metabolite was reflected in the antiviral potency in which $8 \mathrm{mg}$ TAF was similar, and $25 \mathrm{mg}$ TAF was more efficacious in HIV viral load reduction than $300 \mathrm{mg} \mathrm{TDF}^{48} \mathrm{~A}$ recent non-clinical study showed that first-pass hepatic extraction of TAF was $65 \%$ after oral administration suggesting high hepatic uptake. ${ }^{49}$ Since TAF and TDF are similar in 


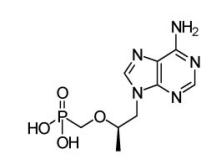

Tenofovir (parent nucleotide)

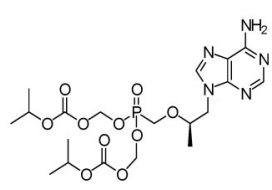

TDF

(DI-ESTER prodrug)

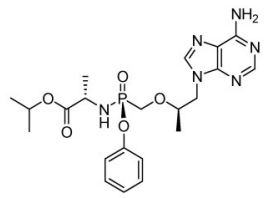

TAF (GS-7340) (MONO-AMIDATE prodrug)

\begin{tabular}{|c|c|c|}
\hline \multirow{2}{*}{ Compound } & \multicolumn{2}{|c|}{ Stability $\mathrm{T}_{1 / 2}(\mathrm{~min})$} \\
\cline { 2 - 3 } & Human plasma & T-cell extract \\
\hline Tenofovir & stable & stable \\
\hline TDF & 0.4 & 71 \\
\hline TAF (GS-7340) & 90 & 28 \\
\hline
\end{tabular}

Fig. 3. TAF is a new prodrug of tenofovir. TDF and TAF are oral prodrugs of tenofovir. Both can be hydrolyzed in serum and are taken up into target cells. However, TAF is more efficient in delivering tenofovir into cells. TAF is more stable in the circulation allowing more efficient transport across cell membrane; it is more readily converted to tenofovir once inside target cells. ${ }^{47}$

anti-HBV potency in cell-based assays, it is possible that compared to TDF, a similar efficacy at lower dose of TAF can be achieved in patients with CHB. This would result in a decrease in systemic exposure of tenofovir in patients.

\section{Expanding access}

To date, more than 4 millions individuals take TDF daily for the treatment of HIV/AIDS and/or hepatitis B. Of note, patients living in the developed world only account for less than 1 million of this population. More than 3 million patients in the developing world receive TDF at no-profit or drastically reduced costs. The distribution to lower-income countries is made possible through a company-run operationally innovative program aimed to provide sustainable access of drugs for life-threatening disease to patients who need them, regardless where they may live. ${ }^{50}$ Such a developing-world access program involves partnerships between international stakeholders such as the patent-holding company, licensed generic drug manufacturers, global health organizations, regional medical communities, health ministries in developing countries, and others.

Nevertheless, only a minute proportion of $\mathrm{CHB}$ patients who are eligible for treatment are currently receiving TDF in lower-income countries in the world. Continuation of efforts and collaborations are much needed to further expand access of effective medications to the needed populations.

\section{Conclusions}

Scientific innovations optimized nucleotide analogs such as tenofovir to be a potent and selective antiviral compound. Medicinal research endeavor transformed tenofovir into TDF for clinical applications, thus advancing the drug to become a critical medicine for treating life-threatening infections suffered by millions worldwide. In order to make a population-wide impact on CHB-associated liver disease, improvements in screening, diagnosis, and access to the knowledge of treatment and prevention are as important as access to the drugs themselves. CHB is a global public health challenge. Support and participation from national governments are much needed to have a significant impact on CHB on a global level.

\section{Conflict of interest}

L. Lou is a former employee of Gilead Sciences, Inc. This review was written without funding support from Gilead, and was entirely self-funded by the author.

\section{Author contributions}

Conceiving and writing the review (LL).

\section{References}

[1] De Clercq E, Holý A, Rosenberg I, Sakuma T, Balzarini J, Maudgal PC. A novel selective broad-spectrum anti-DNA virus agent. Nature 1986;323:464-467.

[2] Della Negra M, de Carvalho AP, de Aquino MZ, da Silva MT, Pinto J, White K, et al. A randomized study of tenofovirdisoproxilfumarate in treatmentexperienced HIV-1 infected adolescents. Pediatr Infect Dis J 2012;31:469473.

[3] Gilead Sciences. Viread (tenofovirdisoproxilfumarate). US prescribing information, August 2012.

[4] Murray KF, Szenborn L, Wysocki J, Rossi S, Corsa AC, Dinh P, et al. Randomized, placebo-controlled trial of tenofovirdisoproxilfumarate in adolescents with chronic hepatitis B. Hepatology 2012;56:2018-2026.

[5] Lok AS, McMahon BJ. Chronic hepatitis B: Update 2009. AASLD Practice Guidelines. Hepatology 2009;50:1-36.

[6] European Association for the Study of the Liver. EASL clinical practice guidelines: management of chronic hepatitis B. J Hepatol 2012;57:167-185.

[7] Liaw Y-F, Kao J-H, Piratvisuth T, Chan HLY, Chien R-N, Liu C-J, et al. AsianPacific consensus statement on the management of chronic hepatitis $\mathrm{B}$ : a 2012 update. Hepatol Int 2012;6:531-561.

[8] Panel on Antiretroviral Guidelines for Adults and Adolescents. Guidelines for the use of antiretroviral agents in HIV-1-infected adults and adolescents. Department of Health and Human Services. March 272012

[9] European AIDS Clinical Society (EACS). EACS guidelines - Version 6.1 November 2012.

[10] Grant RM, Lama JR, Anderson PL, McMahan V, Liu AY, Vargas L, et al. Preexposure chemoprophylaxis for HIV prevention in men who have sex with men. N Engl J Med 2010;363:2587-2599.

[11] Baeten JM, Donnell D, Ndase P, Mugo NR, Campbell JD, Wangisi J, et al. Antiretroviral prophylaxis for HIV prevention in heterosexual men and women. N Engl J Med 2012;367:399-410.

[12] Arimilli MN, Kim CU, Dougherty J, Mulato A, Oliyai R, Shaw J P, et al. Synthesis, in vitro evaluation and oral bioavailability of 9-(2-phosphonomethoxypropyl)adenine prodrugs. Antivir Chem Chemother 1997;8:557567.

[13] Shaw JP, Sueoko CM, Oliyai R, Lee WA, Arimilli MN, Kim CU, et al. Metabolism and pharmacokinetics of novel oral prodrugs of 9-[(R)-2-(phosphonomethoxy)propyl]adenine (PMPA) in dogs. Pharm Res 1997; 14:1824-829.

[14] Robbins BL, Srinivas RV, Kim C, Bischofberger N, Fridland A. Anti-human immunodeficiency virus activity and cellular metabolism of a potential prodrug of the acyclic nucleoside phosphonate 9-R-(2-phosphonomethoxypropyl)adenine (PMPA), Bis(isopropyloxymethylcarbonyl)PMPA. Antimicrob Agents Chemother 1998;42:612-617.

[15] Lee WA, Martin JC. Perspectives on the development of acyclic nucleotide analogs as antiviral drugs. Antiviral Res 2006;71:254-259.

[16] Marcellin P, Heathcote EJ, Buti M, Gane E, de Man RA, Krastev Z, et al. Tenofovirdisoproxilfumarate versus adefovirdipivoxil for chronic hepatitis B.N Engl J Med 2008;359:2442-2455.

[17] Starrett JE Jr, Tortolani DR, Russell J, Hitchcock MJ, Whiterock V, Martin JC et al. Synthesis, oral bioavailability determination, and in vitro evaluation of prodrugs of the antiviral agent 9-[2-(phosphonomethoxy)ethyl]adenine (PMEA). J Med Chem 1994;37:1857-1864.

[18] Kahn J, Lagakos S, Wulfsohn M, Cherng D, Miller M, Cherrington J, et al. Efficacy and safety of adefovirdipivoxil with antiretroviral therapy. JAMA $1999 ; 282: 2305-2312$

[19] Heathcote EJ, Jeffers L, Wright T, Sherman M, Perrillo R, Sacks S, et al. Loss of serum HBV DNA and HBeAg and seroconversion following short-term (12 weeks) adefovirdipivoxil therapy in chronic hepatitis $B$ : two placebocontrolled phase II studies. Hepatology 1998;28(Suppl):317A.

[20] Marcellin P, Chang T-T, Lim SG, Tong MJ, Sievert W, Shiffman ML, J, et al. Adefovirdipivoxil for the treatment of hepatitis $B$ e antigen-positive chronic hepatitis B. N Engl J Med 2003;348:808-816.

[21] Hadziyannis SJ, Tassopoulos NC, Heathcote EJ, Chang TT, Kitis G, Rizzetto M, et al. Adefovirdipivoxil for the treatment of hepatitis $\mathrm{B}$ e antigen-negative chronic hepatitis B.N Engl J Med 2003;348:800-807. 
[22] Cihlar T, Chen MS. Incorporation of selected nucleoside phosphonates and anti-human immunodeficiency virus nucleotide analogs into DNA by human DNA polymerase alpha, beta, and gamma. Antivir Chem Chemother 1997;8: 187-195.

[23] Segovia MC, Chacra W, Gordon SC. Adefovirdipivoxil in chronic hepatitis B: history and current uses. Expert Opin Pharmacother 2012;13:245-254.

[24] Heathcote EJ, Marcellin P, Buti M, Gane E, De Man RA, Krastev Z, et al. Threeyear efficacy and safety of tenofovirdisoproxilfumarate treatment for chronic hepatitis B. Gastroenterology 2011;140:132-143.

[25] Marcellin P, Gane E, Buti M, Afdhal N, Sievert W, Jacobson IM, et al. Regression of cirrhosis during treatment with tenofovirdisoproxilfumarate for chronic hepatitis B: a 5-year open-label follow-up study. Lancet. 2013;381: 468-475.

[26] Pol S, Lampertico P. First-line treatment of chronic hepatitis B with entecavir or tenofovir in "real-life" settings: from clinical trials to clinical practice.J Viral Hepat 2012;19:377-386.

[27] Petersen J, Heyne R, Mauss S, Schlaak J, Schiffelholz W, Eisenbach C, et al. Tenofovir DF for chronic hepatitis B patients in field practice - results from the Geminis German multicenter observational study. J Hepatol 2012;56 (Suppl 2):S212-S213.

[28] Marcellin P, Zoulim F, Causse X, Hezode C, Larrey D, Pageaux G, et al. High efficacy and safety of tenofovir DF in 441 naïve and nuc-experienced chronic hepatitis B patients: A real life multicenter prospective cohort study. J Hepatol 2012:56 (Suppl 2):S210.

[29] Ristig MB, Crippin J, Aberg JA, Powderly WG, Lisker-Melman M, Kessels L, et al. Tenofovirdisoproxilfumarate therapy for chronic hepatitis $B$ in human immunodeficiency virus/hepatitis B virus-coinfected individuals for whom interferon-alpha and lamivudine therapy have failed. J Infect Dis 2002;186: 1844-1847.

[30] Benhamou Y, Tubiana R, Thibault V. Tenofovirdisoproxilfumarate in patients with HIV and lamivudine-resistant hepatitis B virus. N Engl J Med 2003;348: 177-178.

[31] vanBömmel F, Schernick A, Hopf U, Berg T. Tenofovirdisoproxilfumarate exhibits strong antiviral effect in a patient with lamivudine-resistant severe hepatitis B reactivation. Gastroenterology 2003;124:586-587.

[32] van Bömmel F, Zöllner B, Sarrazin C, Spengler U, Hüppe D, Möller B, et al. Tenofovir for patients with lamivudine-resistant hepatitis B virus (HBV) infection and high HBV DNA level during adefovir therapy. Hepatology 2006; 44:318-325.

[33] Lada O, Benhamou Y, Cahour A, Katlama C, Poynard T, Thibault V. In vitro susceptibility of lamivudine-resistant hepatitis B virus to adefovir and tenofovir. Antiviral Res 2004;9:353-363.

[34] Hann H-W, Chae HB, Dunn SR. Tenofovir(TDF) has stronger antiviral effect than adefovir (ADV) against lamivudine (LAM)-resistant hepatitis B virus (HBV). Hepatol Int 2008;2:244-249.

[35] Zoulim F, Locarnini S. Management of treatment failure in chronic hepatitis B. J Hepatol 2012;56(Suppl)1:S112-S122.

[36] Fung S, Kwan P, Fabri MJ, Horban A, Pelemis M, Hann H-W, et al. Efficacy and safety of tenofovir DF in chronic hepatitis $B$ virus infected patients with documented lamivudine resistance. Hepatology 2012;56 (Suppl) S14:200A.
[37] Delaney $W E$, Ray AS, Yang $H$, Qi $X$, Xiong $S$, Zhu $Y$, et al. Intracellular metabolism and in vitro activity of tenofovir against hepatitis $B$ virus. Antimicrob Agents Chemother 2006;50:2471-2477.

[38] Berg T, Marcellin P, Zoulim F, Moller B, Trinh H, Chan S, et al. Tenofovir is effective alone or with emtricitabine in adefovir-treated patients with chronic-hepatitis B virus infection. Gastroenterology 2010;139:1207-1217.

[39] Levrero M, Cimino L, Lampertico P, Viganò M, Gaeta GB, Brancaccio G, et al. OptiB - a multicenter prospective open label study on tenofovir (TDF) for chronic hepatitis B patients with suboptimal response to adefovir (ADV) or ADV/LAM treatment. J Hepatol 2010;52(Suppl 1):S390.

[40] van Bömmel F, de Man RA, Wedemeyer H, Deterding K, Petersen J, Buggisch $\mathrm{P}$, et al. Long-term efficacy of tenofovirmonotherapy for hepatitis $\mathrm{B}$ virusmonoinfected patients after failure of nucleoside/nucleotide analogues. Hepatology 2010;51:73-80.

[41] Patterson SJ, George J, Strasser SI, Lee AU, Sievert W, Nicoll AJ, et al. Tenofovirdisoproxilfumarate rescue therapy following failure of both lamivudine and adefovirdipivoxil in chronic hepatitis B. Gut 2011;60:247-254

[42] Lim LY, Patterson S, Angus PW, Bowden S, Thompson AJ, Locarnini S, et al. Tenofovir rescue therapy achieves long-term suppression of HBV replication in patients with multi-drug resistance HBV: 4 year follow-up of the TDF-109 cohort. Hepatology 2012;56(Suppl)S1:368A.

[43] Neff GW, O'Brien CB, Nery J, Shire N, Montalbano M, Ruiz P, et al. Outcomes in liver transplant recipients with hepatitis $B$ virus: resistance and recurrence patterns from a large transplant center over the last decade. Liver Transpl 2004;10:1372-1378.

[44] Teperman LW, Poordad F, Bzowej N, Martin P, Punpapong S, Schiano T, et al. Randomized trial of emtricitabine/tenofovirdisoproxilfumarate after hepatitis B immunoglobulin withdrawal after liver transplantation. Liver Transpl 2013; 19:594-601.

[45] Liaw Y-F, Sheen I-S, Lee C-M, Akarca US, Papatheodoridis GV, Wong FS-H, et al. Tenofovirdisoproxilfumarate (TDF), emtricitabine/TDF, and entecavir in patients with decompensated chronic hepatitis B disease. Hepatology 2011 . 53:62-72.

[46] Kim WR, Terrault NA, Pedersen RA, Therneau TM, Edwards E, Hindman A, et al. Trends in waiting list registration for liver transplantation for viral hepatitis in the United States. Gastroenterology 2009;137:1680-1686.

[47] Lee WA, He GX, Eisenberg E, Cihlar T, Swaminathan S, Mulato A, et al. Selective intracellular activation of a novel prodrug of the human immunodeficiency virus reverse transcriptase inhibitor tenofovir leads to preferential distribution and accumulation in lymphatic tissue. Antimicrob Agents Chemother 2005;49:1898-1906.

[48] Ruane PJ, DeJesus E, Berger D, Markowitz M, Bredeek UF, Callebaut C, et al. GS-8340 $25 \mathrm{mg}$ and $40 \mathrm{mg}$ demonstrate greater antiviral activity compared with TDF $300 \mathrm{mg}$ in a 10-day monotherapy study of HIV-1 infected patients. 19th Conference of Retroviruses and Opportunistic Infections. 2012:A103.

[49] Babusis D, Phan TK, Lee WA, Watkins WJ, Ray AS. Mechanism for effective lymphoid cell and tissue loading following oral administration of nucleotide prodrug GS-7340. Mol Pharm 2013;10:459-466.

[50] Rangan VK, Lee K. Gilead Sciences, Inc.: Access Program. Harvard Business School Case Study. October 8, 2009. Prod.\#:510029-PDF-ENG. 